\title{
Convergent Validity of Revealed and Stated Recreation Behavior with Quality Change: A Comparison of Multiple and Single Site Demands ${ }^{1}$
}

\author{
John C. Whitehead ${ }^{2}$ \\ Appalachian State University \\ Daniel Phaneuf \\ North Carolina State University \\ Christopher F. Dumas, Jim Herstine, Jeffery Hill, and Bob Buerger \\ University of North Carolina Wilmington
}

December 2007 (revised December 3, 2008)

\footnotetext{
${ }^{1}$ We thank Ju-Chin Huang, two journal referees, and seminar participants at Appalachian State University, University of Delaware, University of Nevada - Las Vegas, University of South Carolina and the National Center for Environmental Economics (USEPA) for many helpful comments on an earlier version of this paper. A previous version of this paper was presented at the 2007 American Agricultural Economic Association Meetings in Portland, OR. This research was partially supported by The U.S. Army Corps of Engineers, Wilmington District, under terms of Contract Number DACW54-03-C-0008.

${ }^{2}$ Contact Author: John C. Whitehead, Department of Economics, Appalachian State University, Boone, NC 28608; email: whiteheadjc@appstate.edu; phone: (828)262-6221.
} 


\section{Convergent Validity of Revealed and Stated Recreation Behavior with Quality Change:}

\section{A Comparison of Multiple and Single Site Demands}

\section{Abstract}

We consider the convergent validity of several demand models using beach recreation data. Two models employ multiple site data: a count data demand system model and the KuhnTucker demand system model. We explore the role of existing variation in beach width in explaining trip choices, and analyze a hypothetical 100 foot increase in beach width. We compare these models to a single equation model where we jointly estimate revealed and stated preference trip data, and focus on a hypothetical scenario considering a 100 foot increase in beach width. In each case we develop estimates of the change in beach visits and the welfare impacts from the increase in width. The trip change estimates from two of the three models are similar and convergent valid, though the willingness to pay estimates differ in magnitude. 


\section{Introduction}

Assessment of the benefits and costs of environmental regulation is often needed to determine the efficiency of policy alternatives. The benefits of environmental quality can be measured with revealed and stated preference approaches. Revealed preference (RP) approaches, such as the travel cost method, relate data on actual choices to observed levels of environmental quality (Phanuef and Smith, 2005). Stated preference (SP) approaches, such as contingent valuation or contingent behavior, use hypothetical choices to measure preferences for a wider range of environmental quality levels (Carson and Hanemann, 2005). In this paper we contribute to the literature comparing estimates for like policy measures obtained from the different approaches.

The travel cost method uses the inverse relationship between trip costs, derived from distance to the recreation site, and visits to estimate recreation demand models. A strength of the travel cost method is that it is based on actual recreational trip behavior. With revealed preference data, individuals consider the costs and benefits of their actions and experience the consequences of their choices. A weakness of the travel cost method for environmental valuation is its reliance on historical data. Proposed policy changes for environmental quality may be beyond the range of historical experience and predictions based on revealed preference models may therefore be limited.

The contingent behavior method is a stated preference approach that can directly elicit recreational trip information from survey respondents based on hypothetical conditions. The method involves the development of a hypothetical situation in which respondents are informed about the status quo and some change away from the status quo. A hypothetical question is presented that confronts respondents with a choice about behavior under the new environmental 
quality (and/or changed costs) vis-à-vis the status quo. A feature of the contingent behavior approach is its flexibility. Hypothetical choices may be the only way to gain policy relevant information when historic variability in environmental conditions is limited. A weakness of the contingent behavior approach is its hypothetical nature. Respondents are placed in unfamiliar situations in which complete information is not available. Respondents may discount costs or income constraints or optimistically forecast avid recreation behavior.

The combination and joint estimation of revealed and stated preference data exploits the contrasting strengths of the travel cost method and contingent behavior (see Whitehead et al., 2008 for a review of this literature). Combining SP data with RP data grounds hypothetical choices with real choice behavior and may improve forecasts beyond the range of historical experience. Continuous choice RP and SP data have a common structure and can be stacked and jointly estimated. For example, Layman, Boyce and Criddle (1996) and Eisworth et al. (2000) pool the data and estimate models assuming the errors are independently and identically distributed. Englin and Cameron (1996) compare the pooled data model with a fixed effects model, treating the data as a panel, and find few differences. Whitehead, Huang, and Haab (2000) and Azevedo, Herriges, and Kling (2003) treat the data as a panel and use random effects Poisson and Tobit models, respectively.

With all valuation methods the goal is to estimate the true benefits of environmental quality, though it can be difficult to recognize when estimates are indeed unbiased. Comparison of nonmarket valuation estimates to a known true value is a construct validity test. Construct validity tests in the environmental valuation literature can be conducted using experimental methods and with the happenstance of natural experiments. In the contingent valuation literature, a large number of construct validity studies compare actual willingness to pay obtained from 
laboratory and field experiments with hypothetical willingness to pay obtained from contingent valuation surveys. Convergence in actual and hypothetical willingness to pay is evidence of construct validity. List and Gallet (2001) perform a meta-analysis of these studies. They find that private goods generate better convergence than public goods, as do SP scenarios based on familiar behavior (e.g. behavior that leads to use value). These results suggest that stated behavior responses should have greater predictive validity than stated willingness to pay responses, since willingness to pay data may confound use and nonuse values. Little and Berrens (2004) expand the List and Gallet sample to include studies with incentive compatible contingent valuation questions and hypothetical bias correction methods; they find no evidence that private goods generate better convergence than public goods, and show that referendum formats and certainty corrections increase convergence.

Construct validity tests outside field and laboratory experiments are rare, with only two reported to our knowledge. Grijalva et al. (2002) survey rock climbers about their past trip behavior and hypothetical behavior under future access conditions. Following the actual closure of rock climbing areas, respondents are surveyed again to determine if their hypothetical choices predict actual behavior under the altered conditions. Whitehead (2005) surveys respondents about their past hurricane evacuation behavior after low-intensity storms and hypothetical behavior with low- and high-intensity storms. Two hurricanes followed the survey and respondents were contacted again to determine their actual evacuation behavior. Both studies find some evidence of construct validity with jointly estimated revealed and stated preference models. While an independently estimated revealed preference model could not be used to make these predictions, left unresolved is the ability of the stated preference data alone to make accurate forecasts. 
When no value for the true theoretical construct of willingness-to-pay exists, convergent validity tests involve comparisons between different nonmarket valuation estimates (Carson et al., 1996). For example, a consumer surplus estimate of recreation benefits derived from the travel cost method can be compared to a willingness to pay estimate of site access derived from the contingent valuation method. If the estimates converge, or diverge for expected reasons, then there is increasing confidence in both approaches. Jeon and Herriges (2005) conduct a convergent validity test for revealed and stated trip behavior with a multiple site demand model. Comparing lake recreation trip predictions based on existing variation in water quality with stated preference trips following a hypothetical improvement in water quality, they find a lack of convergent validity. The revealed preference data predicts a larger trip change than the stated preference data.

In this paper we examine beach recreation behavior using several models and through these models address several issues. We compare two models that use multiple site revealed preference data: a count data demand system model, and the Kuhn-Tucker (KT) demand system model. Both models provide a characterization of seasonal preferences for the beaches and their attributes. We exploit the existing variation in beach width in our choice set to analyze the behavioral and welfare effects of a 100 foot increase in beach width across all sites in the study area. We compare these models to a single equation model where we jointly estimate aggregate trip demand using RP data and SP data describing responses to a hypothetical 100 foot increase in beach width. In each case we develop estimates of the increased number of beach trips from an increase in beach width, and the non-market value of the increase. We assess the convergent validity of trip predictions and welfare effects from the three approaches. 


\section{Data}

The study area includes beaches in five southern North Carolina counties (Figure 1). Bogue Banks, a barrier island, is located in Carteret County, and encompasses a twenty-four mile stretch of beach communities. Topsail Island, a barrier island, is located in both Pender and Onslow Counties and contains a twenty-two mile stretch of beach communities. New Hanover County includes a thirteen mile stretch of beach communities lying between Pender and Brunswick Counties. The Brunswick County beaches are located between the Cape Fear River and the South Carolina border and encompass a twenty-four mile stretch of beach communities.

We use beach recreation data from a recent US Army Corps of Engineers (USACE) funded study (Herstine et al., 2005). The target survey population was chosen based upon the results of an on-site survey conducted during the summer of 2003 at the study area beaches. One finding from the on-site survey is that 73 percent of day users traveled 120 miles or less to get to the beach. For this study, day users are defined as those who leave their home, visit the beach, and return home afterwards without spending the night. Overnight users spend at least one night away from home. Locals are those who live within walking or biking distance of the beach.

A telephone survey of all types of beachgoers who traveled 120 miles or less to get to the beach - day users, overnight users and locals - was administered by the Survey Research Laboratory at the University of North Carolina at Wilmington. Survey Sampling, Inc. provided telephone numbers within the study area. The telephone survey was conducted during May 2004 and a 52 percent response rate was realized.

Our analysis sample was determined as follows. Among the telephone survey respondents 1509 stated that they had considered going to an oceanfront beach in North Carolina during the last year (2003). Of this number, 1375 completed the survey; removal of ineligible 
respondents (e.g., younger than 18 years) reduced the sample to 1276 . Deleting responses with incorrect zip codes (and therefore missing distance variables required to calculate travel costs), missing income and demographics, or those living further than 155 miles from a site reduced the sample to 1112. Removing respondents with missing revealed preference trip data reduces the sample to 868 . Removing people who report taking more than one hundred trips in the season and deleting respondents with missing stated preference trip data further reduces the core sample to 638 .

In order to enhance comparability between the multiple site and single site data we delete cases for which the sum of the trips from the multiple site data is not equal to the aggregate number of trips from the single site data. These data may not be equal for a variety of reasons. Respondents may suffer from recall error with multiple site or aggregate beach trip questions. Also, respondents may report trips taken to beaches other than one of the 17 study sites or fail to report trips to all 17 study sites when reporting aggregate beach trips. Our final analysis sample size is 419. Summaries for observable socioeconomic factors do not differ between respondents included in the analysis sample and those excluded. ${ }^{1}$

Approximately $80 \%$ of the respondents stated that 2003 was a typical year in terms of their oceanfront beach trips to the southern NC coast. Of those who reported that 2003 was not a

\footnotetext{
${ }^{1}$ Selection effects are certainly a concern given how our analysis sample was obtained. To address this, we have analyzed the various subsamples of data and compared consumer surplus estimates and trip predictions for beach width change scenarios using single-site and multi-site models. Trip predictions and welfare measures do not differ substantially across the subsamples. While selection effects may still remain, their importance is likely small for our model comparison (as opposed to population prediction) objectives. By accepting the risk of selection bias, we eliminate confounding effects related to recall or other errors. The results from our auxiliary analyses are available upon request.
} 
typical year in terms of oceanfront beach trips to the southern NC coast, $75 \%$ would normally have taken more trips. Of all respondents who took at least one trip to the southern $\mathrm{NC}$ coast, 96\% planned to take at least one oceanfront beach trip to this area in 2004.

The telephone survey elicited information on whether respondents took day trips only or a mix of day and overnight trips. Thirty-eight percent of the sample took only day trips. The problem with multiple purpose trips is that the willingness to pay for the recreation trip or a characteristic of the trip may be biased. The bias may be positive if the beach trip is a minor reason for taking the overnight trip. For example, vacationers may spend more time at an amusement park or shopping than at the beach. There exists a variety of approaches to overnight trips (Parsons 2003). Since we are unable to distinguish between day trips and overnight trips, we pool the data. In our modeling we assume that beach recreation is the primary purpose of the trip and attribute all of the willingness to pay to that purpose. Since our objective is to compare estimates across models, the inclusion of multiple purpose overnight trips should have little impact.

The single site revealed preference beach trips were elicited by asking respondents who had actually taken oceanfront beach trips to the North Carolina coast in 2003 how many of their oceanfront beach trips were to the southern NC coast from the Beaufort/Morehead City area in Carteret County to the South Carolina border. The number of annual trips ranges from 0 to 100 and the mean annual number of trips is nearly 8 (see Table 1). Respondents who planned to take at least one oceanfront beach trip to the southern NC coast during 2004 were asked how many trips they would take. The average number of planned trips with current quality conditions is 10 . Improved access conditions are described as "no time spent searching for a parking spot, reasonable fees, and no change in congestion.” The average number of planned trips with 
improved access conditions is 14. Increased beach width is described as "adding an average of 100 feet to beach width with periodic beach nourishment every 3 to 5 years." The average number of planned trips with increased beach width is 11 .

Travel distances and time between each survey respondent's home zip code and the zip code of the population center of each beach county were calculated using the ZIPFIP correction for "great circle" distances (Hellerstein et al. 1993). Additional distance to each beach town was calculated using Mapquest. Travel time was calculated by dividing distance by 50 miles per hour. The cost per mile used was $\$ 0.37$, the national average automobile driving cost for 2003 including only variable costs and no fixed costs as reported by the American Automobile Association (AAA, 2005). Thirty-three percent of the wage rate was used to value leisure time for each respondent. The round-trip travel cost is $p=(2 \cdot c \cdot d)+(\theta w) \cdot(2 \cdot d m p h)$, where $c$ is cost per mile, $d$ is one-way distance, $\theta$ is the fraction of the wage rate, $w$, and $m p h$ is miles per hour. We use household income divided by 2000 hours to estimate the household wage. In Table 1 we report the travel cost associated with the distance for each household to the most visited beach among the 17 beaches in the study site. When there are two or more beaches that are visited equally, the distance is chosen randomly among the subset of beaches.

The beach site characteristics data and visitation frequency are presented in Table 2. The most popular beaches are Atlantic Beach, Emerald Isle and Wrightsville Beach. Beach characteristic data include beach width, beach length, the number of parking spaces and the number of public access points. Average beach length was found using various USACE project books. Parking access points and parking spaces were also collected from USACE project data. Average beach width was estimated using USACE aerial photography from 2002 and was from the mean high water line to the first line of vegetation. The average beach width is 130 feet, the 
minimum beach width is 80 feet (Caswell Beach), and the maximum width is 400 feet (Fort Fisher).

\section{Recreation Demand Models}

Single Equation Revealed-Stated Preference Model

Consider a single-site recreation demand model with revealed and/or stated preference data. A common functional form for the single-site demand model is the semi-log

$$
x=\exp \left(\beta_{0}+\beta_{1} p+\beta_{2} y\right)
$$

where $x$ is the number of trips, $p$ is the own-price (i.e., round trip travel costs to the beach site), $y$ is income and $\beta_{0}, \beta_{1}, \beta_{2}$ are coefficients. The intercept term may implicitly or explicitly include site quality or respondent characteristic variables. We estimate a version of (1) using RP and SP data. Revealed preference information on total trips to beaches in our study region at status quo conditions is available for all respondents in our analysis sample. The same people answered stated preference trip questions about future trips: (a) under status quo conditions, (b) with an improvement in access conditions, and (c) with an increase in beach width.

In our most general models we pool the data for all individuals and responses. In these cases we use panel methods to account for the separate variances across individuals and scenarios. In particular, we estimate equation (2) with the random effects Poisson model that includes a stated preference dummy variable and interaction terms:

$$
\begin{aligned}
\ln \mu_{i t}=\ln \lambda_{i t}+u_{i}=\beta_{0} & +\beta_{1} p_{i}+\beta_{2} y_{i}+\beta_{3} q_{3}+\beta_{4} q_{4} \\
& +\delta_{1} S P+\delta_{2}\left(S P \times p_{i}\right)+\delta_{3}\left(S P \times y_{i}\right)+u_{i},
\end{aligned}
$$

where $i$ denotes a respondent, $t=1, \ldots, 4$ indicates alternative trip decision situations in the pseudo-panel data, $q_{3}\left(q_{3}=1\right.$ when $\left.t=3\right)$ and $q_{4}\left(q_{4}=1\right.$ when $\left.t=4\right)$ are beach access and width scenario variables, and $u_{i}$ is the random effect for group (person) $i$. The $S P$ dummy variable and 
interaction variables are included to test for shift and slope differences in $R P$ and $S P$ data, where $S P=1$ for hypothetical trip data $(t=2,3$ or 4$)$ and 0 for revealed trip data $(t=1)$.

Single-site demand models are typically estimated with the Poisson or negative binomial count data regression models, which account for the integer nature of trips (Haab and McConnell 2002). In our model, the distribution of trips $x_{i t}$ conditioned on $u_{i}$ and the covariates is Poisson with conditional mean and variance $\mu_{i t}$. By mixing a person-specific draw from a gamma distribution into the conditional means (i.e. $\exp \left(u_{i}\right)$ is distributed gamma), the random effects Poisson model implies the demand for trips is negative binomial, but that correlation is induced across the panel. In this sense, specifications using single and multiple elements from the panel are comparable (Cameron and Trivedi 1998).

With estimates of the parameters for the demand equation in hand we are interested in predicting the change in trips resulting from a change in beach width, and the welfare effects of this change. To assure comparability with the welfare estimates arising from our demand system models, for the latter we use the exact welfare formulas implied by the semi-log functional form. For the specific case of equation (2) the expected quasi-indirect utility function is

$$
E\left(V_{i}\right)=-\frac{\exp \left(-\beta_{2} y_{i}\right)}{\beta_{2}}-\frac{\exp \left(\beta_{0}+\beta_{1} p_{i}+\beta_{3} q_{3}+\beta_{4} q_{4}\right)}{\beta_{1}},
$$

where for exposition the $S P$ dummy variable has been set to zero, and $\exp \left(u_{i}\right)$ is set to its mean of one. Equation (3) allows us to conduct exact welfare analysis. In particular, person i's expected compensating variation, $C V$, for a change in price or non-price attributes is given by

$$
E\left(C V_{i}\right)=\frac{1}{\beta_{2}} \ln \left[1-\frac{\beta_{2}}{\beta_{1}}\left(E\left(x_{i}^{1}\right)-E\left(x_{i}^{0}\right)\right)\right],
$$

where 


$$
E\left(x_{i}^{k}\right)=\exp \left(\beta_{0}+\beta_{1} p_{i}^{k}+\beta_{2} y_{i}+\beta_{3} q_{3}^{k}+\beta_{4} q_{4}^{k}\right), \quad k=0,1,
$$

and $k=0$ and $k=1$ denote status quo and changed conditions, respectively. ${ }^{2}$

\section{Count Data Demand System}

Consider a multiple site situation in which a person has available $J$ recreation sites and makes decisions on the number of trips to make to each site over the course of a season. We once again use the semi-log form for the demand equations, given in general by

$$
x_{j}=\exp \left(\beta_{j}+\sum_{k=1}^{J} \beta_{j k} p_{j}+\beta_{y j} y\right), \quad j=1, \ldots, J
$$

where $p_{j}$ is the travel cost to site $j, y$ is annual income, and the intercept can contain site quality or individual characteristic variables. In order for (6) to be consistent with a rational preference ordering (the so-called integrability conditions) restrictions on the parameters are necessary (see von Haefen, 2002, for a full discussion). In particular, the restrictions $\beta_{j k}=0$ for $k \neq j$ and $\beta_{y j}=\beta_{y}$ for all $j$ must be imposed such that the demand equation is given by

$$
x_{j}=\exp \left(\beta_{j}+\beta_{j j} p_{j}+\beta_{y} y\right), \quad j=1, \ldots, J .
$$

In our empirical modeling we specify $\beta_{j}$ as a linear combination of site quality attributes such that $\beta_{j}=\beta_{0}+\gamma q_{j}$, where $q_{j}$ is a vector of attributes for site $j$.

To make the model operational we assume trips to each site follow an independent, negative binomial distribution in which the conditional mean $\mu_{i j}$ for visits to site $j$ by person $i$ is

$$
\ln \mu_{i j}=\ln \left(\lambda_{i j}+u_{i j}\right)=\beta_{j}+\beta_{j j} p_{i j}+\beta_{y} y_{i}+u_{i j}, \quad j=1, \ldots, J,
$$

where $u_{i j}$ is a random variable in which $\exp \left(u_{i j}\right)$ is distributed gamma. With the semi-log,

\footnotetext{
${ }^{2}$ This expression is found by implicitly defining $C V_{i}$ via $V_{i}\left(p_{i}^{0}, q_{3}^{0}, q_{4}^{0}, y_{i}\right)=V_{i}\left(p_{i}^{1}, q_{3}^{1}, q_{4}^{1}, y_{i}-C V_{i}\right)$ using (3), and solving out for $C V_{i}$ to arrive at (4). See Freeman (2003, p.70) for discussion of this strategy for 'exact' welfare analysis.
} 
negative binomial assumptions for our demand equations the count data demand system specification is parallel to that used in the single equation analysis. Estimation by maximum likelihood over the $N$ respondents and $J$ equations provides a characterization of the demand equation parameters. $^{3}$

With the demand equation parameters in hand it is possible to write the expected indirect utility function for person $i$ as

$$
E\left(V_{i}\right)=-\frac{\exp \left(-\beta_{y} y_{i}\right)}{\beta_{y}}-\sum_{j=1}^{J} \frac{\exp \left(\beta_{j}+\beta_{j j} p_{i j}\right)}{\beta_{j j}}
$$

In a generalization of equation (4) the expected compensating variation for a change in price or quality terms is

$$
E\left(C V_{i}\right)=\frac{1}{\beta_{y}} \ln \left[1-\frac{\beta_{y}}{\beta_{j j}} \sum_{j=1}^{J}\left(E\left(x_{i j}^{1}\right)-E\left(x_{i j}^{0}\right)\right)\right]
$$

where

$$
E\left(x_{i j}^{k}\right)=\exp \left(\beta_{j}^{k}+\beta_{j j} p_{i j}^{k}+\beta_{y} y_{i}\right), \quad k=0,1, \quad j=1, \ldots, J,
$$

and $k=0$ and $k=1$ again denote status quo and changed conditions, respectively.

\section{Kuhn-Tucker Model}

Consider again a multiple-site recreation setting, but suppose now that people simultaneously decide which sites to visit and how many trips to make to each over the course of a season. The Kuhn-Tucker (KT) demand model (Phaneuf et al. 2000; von Haefen et al. 2004) models this behavior as stemming from a single utility maximization problem. It therefore

\footnotetext{
${ }^{3}$ Estimation is straightforward given the assumption that the $J$ count random variables are independent within and between individuals. A more general mixing distribution allowing correlation results in a more complicated model that requires simulation for estimation. See Eagan and Herriges (2006) for examples of these types of models.
} 
provides a theoretically-consistent approach to describing the combination of interior and corner solutions that are an empirical regularity in multiple site recreation data. The model is, however, computationally more demanding than those outlined above; we thus provide in this section only an overview of its main components. A detailed technical description is given in von Haefen and Phaneuf (2005), and a tutorial-style discussion is provided by Phaneuf and Sideralis (2003).

The model begins with the specification of the consumer's direct utility function $U(\mathrm{x}, q, z, \beta, \varepsilon)$ where $x$ is a $J$-dimensional vector of visits to a set of available recreation sites, $q$ is an $L X J$ matrix of site-specific quality attributes for the recreation sites, $z$ is a strictly positive numeraire denoting spending on all other goods, $\beta$ is a vector of utility function parameters, and $\varepsilon$ is a $J$-dimensional vector of errors. The consumer maximizes utility subject to the budget constraint and non-negativity constraints:

$$
\max _{x, z} U(x, q, z, \beta, \varepsilon) \text { s.t. } y=p^{\prime} x+z, x_{j} \geq 0, j=1, \ldots, J,
$$

where $p$ is the vector of travel costs to each of the available sites and $y$ is the consumer's annual income. The first-order Kuhn-Tucker conditions that characterize the optimal solution to this problem are given by

$$
\begin{gathered}
\frac{\partial U / \partial x_{j}}{\partial U / \partial z} \leq p_{j}, \quad j=1, \ldots, J, \\
x_{j} \times\left[\frac{\partial U / \partial x_{j}}{\partial U / \partial z}-p_{j}\right]=0, \quad j=1, \ldots, J .
\end{gathered}
$$

Equation (13) is central to both the estimation and welfare calculation strategies employed in this model. With assumptions on the functional form for utility and the distribution of the error terms, the $J$ weak inequalities in (13) can be used to specify the probability of observing a particular individual's choice outcomes, and maximum likelihood used to recover estimates of the utility function parameters. 
Estimation of the structural parameters of $U(\cdot)$ provides a characterization of preferences that can be used to calculate Hicksian welfare measures for a change in prices and/or quality levels. In general the compensating variation for a change in quality from baseline conditions $q^{0}$ to a new level denoted $q^{1}$ is given by

$$
C V=y-e\left(p, q^{1}, U^{0}, \beta, \varepsilon\right)
$$

where $e(\cdot)$ is the expenditure function and $U^{0}$ is the baseline level of utility.

In equation $(14) e(\cdot)$ is an endogenous regime switching function in which the regimes correspond to each of the $2^{J}$ possible combinations of interior and corner solutions for the $J$ sites. In addition the error terms $\varepsilon$ are not observed and thus $e(\cdot)$ is not fully known by the analyst, implying $C V$ is a random variable from the analyst's perspective. The inference goal is therefore to calculate the expectation of compensating surplus, denoted $E(C V)$. This requires Monte Carlo integration techniques in which multiple realizations of the errors are simulated and $C V$ calculated conditional on each simulated value. These two aspects of (14) imply that calculating welfare effects (and predicting new trip totals) for counterfactual scenarios involves non-trivial computational challenges. Von Haefen et al. (2004) and von Haefen and Phaneuf (2005), however, describe efficient algorithms for conducting this computation for versions of the utility function that we employ in this paper.

The specific parameterization of the utility function that we use in our analysis is given by:

$$
\begin{aligned}
U(\cdot) & =\sum_{j=1}^{J} \Psi_{j} \ln \left[\phi_{j} x_{j}+\theta\right]+\frac{z^{\rho}}{\rho} \\
\ln \Psi_{j} & =\alpha_{j}+\delta^{\prime} s+\mu \varepsilon_{j} \\
\ln \phi_{j} & =\gamma^{\prime} q_{j} \\
\rho & =1-\exp \left(\rho^{*}\right) \\
\ln \mu & =\mu^{*}
\end{aligned}
$$


where $s$ is a vector of household characteristics, $q_{j}$ is a vector containing the $L$ quality attributes for site $j,\left(\alpha_{j}, \delta, \gamma, \theta, \rho^{*}\right)$ are the structural parameters to be estimated and $\mu$ is a scale parameter common to all the errors. Maximizing (15) with respect to the budget constraint and nonnegativity constraints implies a set of first order conditions that, following some manipulation, can be written as

$$
\varepsilon_{j} \leq \frac{1}{\mu}\left(-\alpha_{j}-\delta^{\prime} \mathbf{s}+\ln \frac{p_{j}}{\phi_{j}}+\ln \left(\phi_{j} x_{j}+\theta\right)+(\rho-1) \ln \left(y-\mathbf{p}^{\prime} \mathbf{x}\right)\right), \quad j=1, \ldots, J .
$$

If each element of $\varepsilon$ is an independent draw from a type I extreme value distribution with scale parameter $\mu$ then equation (16) can be used to state a closed form expression for the probability of observing an individual's trip-taking outcomes. In particular the likelihood of observing a person's outcome $\mathbf{x}$ conditional on the structural parameters is

$$
l\left(\mathbf{x} \mid \delta, \gamma, \theta, \rho^{*}, \mu^{*}\right)=|\mathbf{J}| \prod_{j}\left[\exp \left(-g_{j}(\cdot)\right) / \mu\right]^{1_{x_{j}>0}} \times \exp \left[-\exp \left(-g_{j}(\cdot)\right)\right],
$$

where $g_{j}(\cdot)$ is the right hand side of $(16),|\mathbf{J}|$ is the determinant of the Jacobian of transformation, and $1_{x_{j}>0}$ is an indicator function equal to one if $x_{j}$ is strictly positive and zero otherwise. Equation (17) can be used to form the sample likelihood, and standard maximum likelihood search algorithms used to estimate the structural parameters.

Once the parameters of the utility function are estimated welfare calculation and trip prediction can proceed using the techniques described in von Haefen et al. (2004) and von Haefen and Phaneuf (2005). We follow emerging consensus for these models and apply von Haefen's (2003) conditional welfare measurement approach. This approach implies that, when simulating unobserved heterogeneity from a model with unobserved components, the errors should be drawn conditionally to replicate the observed behavior at baseline conditions. This 
notion places specific ranges of support on the values that individual-level unobserved effects can take, and makes greater use of the information in the sample. Additional details on welfare calculation and prediction for this application are given in the appendix.

\section{Empirical Results}

\section{Parameter Estimates}

Four single equation recreation demand models are estimated with the pseudo-panel data, and results are shown in the top part of Table 3 . The first is the RP model estimated with the negative binomial. The second is the SP model estimated without the RP data. The third and fourth are jointly estimated RP-SP models. The latter three are estimated as random effects Poisson models which correct for over-dispersion and allow correlation across the panel (Haab and McConnell, 2002). In each model the coefficient on the own-price variable is negative and statistically significant and the coefficient on the income variable is positive and statistically significant. The household characteristics variables are generally, but not always, significant across the models. There are no obvious quantitative differences between the independently estimated RP and SP models amongst these standard variables. Finally, in the SP model the coefficients on the improved access and the increase in beach width variables act as demand shifters in the expected direction.

The jointly estimated RP-SP model is almost identical to the SP model with a statistically significant coefficient on the SP dummy variable. This indicates that respondents state that they will take more trips than the revealed preference data indicate. One interpretation of this result is the stated preference data exhibit hypothetical bias; respondents state that they will take more trips than their budget constraints suggest. The final model in Table 3 supports this interpretation. Another interpretation is that the RP data were collected in an unusual year in 
which fewer trips were taken. Some survey evidence supporting this second interpretation was noted in the data section. The final RP-SP model tests for differences in slope coefficients with interaction effects between the SP dummy variable and own-price, income, and the household variables. We find that the SP demand data is less income elastic (income elasticity of 0.98 vs. 0.81 ) suggesting that SP responses pay less attention to income constraints. The travel cost elasticity of -0.89 is essentially equal for the RP and SP demand data. ${ }^{4}$

Selected parameter estimates for four specifications of the count data demand system model are shown in Table 4, and the full set of estimates (including all price parameters) are shown in the Appendix. Models 1 and 2 are our full specifications in that they include all four site attributes as well as dummy variables for Fort Macon and Fort Fisher, which are differentiated by their status as state parks of historic interest. The two full specifications are distinguished by the use of level width in model 1 and log width in model 2. Models 3 and 4 maintain the log width transformation while dropping the length and parking attributes, respectively.

Two observations emerge from these models as well as several others examined but not reported. First, and most obviously, we find essentially no evidence that beach width affects trip demand among the sampled individuals. Instead price and income effects dominate, and in some models other attributes are marginally significant. The income elasticity for all sites is 1.16 , and the price elasticities computed at the data averages range from -4.56 to -0.39 (the median for the 17 sites is -2.22). These findings are consistent across specifications and hold for Poisson models as well (which are more robust to misspecification of the conditional mean). Second, and related

\footnotetext{
${ }^{4} \mathrm{We}$ also include SP interaction terms with the married and children variables. SP responses are less responsive to demographic constraints. The SP interaction variables are jointly significant according to the LR test.
} 
to this, the level and log specifications for width have different behavioral implications, but we find little practical difference between models using one or the other. We focus on the log specifications here and subsequently, since it seems intuitive that the behavioral effect of increased beach width is not monotonically increasing. Taken together the results from our count system models imply that the multiple site RP data as used with this model do not replicate the qualitative findings from the SP analysis.

The KT model estimation results are shown in Table 5. We report four specifications that match those used in the count system models in terms of how site and household characteristics enter the models. We note, however, that the KT model is highly non-linear in its parameters and comparisons of coefficient magnitudes across the two multiple site models are not appropriate. All the models are estimated with fixed effects (separate $\alpha_{j}^{\prime}$ s) for each of the 17 beaches, thereby providing some accounting for unobserved site attributes. As with our count specifications, we focus primarily on the log specification for beach width.

The estimates for the utility function and error variance parameters $\left(\theta, \rho^{*}, \mu^{*}\right)$ are significant, appropriately signed, and similar across the different model specifications. The household variables generally provide little explanatory power, though their signs match what was found in the other models. The fixed effects function as site specific intercepts that enter through the $\Psi_{j}(\cdot)$ terms in equation (15). In contrast to the count demand system model, these provide some accounting for unobserved site attributes. Of more direct interest are the estimates for the observed site attributes. Unlike the count system, we find a positive effect for beach width that is robust across model specifications. The size of the effect is relatively stable, though it does depend to some degree on which of the other site attributes are included in the specification. Surprisingly, the other site attributes are generally not significant determinants of behavior. 
Nonetheless the robustly positive and significant coefficient on beach width suggests that, when the KT multiple site RP model is used, we are able to replicate the qualitative findings from the combined SP-RP model.

\section{Welfare and Trip Predictions}

To conduct our convergent validity analysis with more formality we examine trip predictions and welfare effects from each modeling approach for a common policy scenario. Point estimates for seasonal welfare effects and trip changes from a 100 foot increase in width at all beaches in the study area are shown for all specifications of our single equation, count system, and KT models at the bottom of Tables 3, 4, and 5, respectively. In Table 6 we show a comparison of estimates and standard errors across the three approaches, using in each case a preferred specification. All welfare estimates are individual, exact compensating variation measures for the recreation season. Changes in trips are aggregates for all seventeen sites in the study area.

Focusing first on the preferred specifications in Table 6, we find that the KT and RP-SP models provide similar and statistically indistinguishable predictions for the change in trips. On average according to these models, respondents will take 1 extra trip per season as a result of the increased beach width. Our estimates of welfare effects are roughly similar in their order of magnitude and statistically similar due to the comparatively noisy estimates from the KT model. The size of the point estimates ( $\$ 106-\$ 126$ vs. $\$ 309)$, however, are different enough to imply economically meaningful differences in the welfare predictions. Thus we conclude that the KT and RP-SP models are convergent valid in trip prediction, but convergent invalid in welfare 
effects. $^{5}$

A different story emerges for the count system model. Having found no effect from beach width we do not find economic or statistically significant changes in trip demand from the increased beach width. From this we conclude that the count model is convergent invalid with both the KT and RP-SP models.

As a final note we consider the convergent validity of the SP and RP-SP versions of the single equation model. The point estimates for trip changes and welfare effects shown at the bottom of Table 3 suggest that quite similar results emerge from the analysis regardless of the inclusion of the RP data. The comparison with standard errors in Table 6 confirms that the estimates are statistically indistinguishable. Thus, for analyzing the quality change, the SP and RP-SP models are convergent valid. We note, however, that the larger number of predicted trips in the SP-only model implies a larger total value of beach access compared to the RP-only and RP-SP models.

The ultimate use of estimates as described here lies in policy analysis. Though the KT and RP-SP models are convergent valid in some dimensions, their willingness to pay estimates show differences that are likely economically significant. As an illustration consider a comparison of the recreation benefits and costs of beach nourishment. With 1.58 million

\footnotetext{
${ }^{5}$ An earlier version of this paper included a linked nested logit-aggregate trip frequency negative binomial model estimated with the RP data (see Parsons et al. 1999 for a description). This model also predicted respondents would take one additional trip per year following a 100 foot increase in beach width across all 17 beach sites. The annual willingness to pay for the improvement of approximately $\$ 32$ was notably smaller than that obtained from the KT and RP-SP models. This suggests trip predictions were convergent valid between the linked, KT, and RP-SP models but convergent invalid in welfare measurement. The linked model was ultimately removed from the paper due to its ad hoc nature and incomparability of welfare effects with the structural models.
} 
households in the study region, the aggregate annual recreation benefit is $\$ 167$ million and $\$ 488$ million when estimated with the single equation and Kuhn-Tucker models, respectively. A rule of thumb is that the annual cost to replace one foot of eroded beach is $\$ 32,000$ per mile. In the study region there are 60 miles of beach length. Adding 100 feet every 4 years would cost $\$ 48$ million annually. The annual net benefits of beach nourishment range from $\$ 119$ million to $\$ 440$ million. Use of either model would suggest that beach nourishment is an efficient policy. However, in a benefit cost analysis with other beach management alternatives relative to the status quo (e.g., beach hardening, beach retreat), the choice of recreation demand model could have implications for the preferred policy alternative.

\section{Conclusions}

This paper has compared three types of models using a rich beach recreation data set for southern North Carolina. In general we find that the models provide reasonable descriptions of recreation behavior, though they vary in the extent to which site attributes explain observed choices. In an analysis of the benefits of increased beach width we find plausible and significant welfare measures for two of the three approaches examined. A problem for policy application is choosing amongst different welfare measures. When estimates from different models yield convergent results (or diverge for known reasons) there is increased confidence in the suitability of estimates for meaningful policy inference.

Our assessment of the convergent validity of the three models examined is mixed. Trip predictions from the RP-SP and KT models (and the unreported linked model) are convergent valid. Welfare measures, while similar statistically and in order of magnitude, are economically different. Thus these models converge in behavior predictions but not in willingness to pay estimates. Nonetheless for many policy applications similar inference may result from the two 
models. For the count data demand system models no significant effect was found for our policy attribute, and as such predictions from this model are convergent invalid with both the KT and RP-SP models.

What explanations can be given for these findings? Three observations are relevant for answering this. First, the sources of variability used in the single equation and multiple site models are quite distinct. In the single equation analysis identification of the width effect comes from the SP design, and the relevant behavioral margin is aggregate trip frequency as it responds to an experimentally designed discrete change in width. In the multiple site models identification of the width effect is off of the observed variability in beach width across the seventeen beaches in the study region. The behavioral margin responding to beach width variability is trip frequency and site choice.

This leads to the second observation, which concerns how the multiple site models exploit the natural variation in beach width. The count demand system model fits an average interior solution for the system of $J$ equations (recall all expected demands are strictly positive). While the negative binomial distribution accommodates zero valued outcomes, the underlying model does not address the extensive margin decision over which set of sites to visit. This implies that the count system model identifies the beach width effect only by differences in trip frequency among the several sites. The KT model in contrast uses information on both trip frequency and site choices to capture the role of beach width and other attributes. The robustly positive effect from the KT models and absence of an effect from the count data models suggest the site choice aspect of visit decisions is perhaps more relevant for understanding the role of beach width using multiple site models. 
Our third observation relates to how the individual models control for confounding effects. The single equation model depends on the SP design, which asks respondents to answer questions while cogitatively holding all else fixed. This assumption is not tenable in the RP data, where outcomes are driven by many factors, only some of which can be objectively measured. The linearity (in logs) of the count demand specification implies one cannot include site specific constants and site attributes that vary only across sites. This suggests that omitted attributes that are correlated with an attribute of interest (i.e. beach width) can generate biased estimates. The KT model, primarily via its non-linearity, allows inclusion of fixed effects and site attributes in different sub-functions of the utility specification. Though certainly not an ideal solution to the omitted attributes problem, this suggests the KT model may be more robust to misspecification vis-à-vis the count data model.

These observations lead us to conclude that the KT and count data demand system models diverge for plausible reasons in this application, suggesting that some confidence can be taken from the comparison of trip predictions from the RP-SP and multiple equation models. Nonetheless differences in willingness to pay estimates remain.

Our analysis raises a number of issues. First, combined with the evidence of Jeon and Herriges (2005), the typical concern with contingent behavior data that respondents will overstate trip taking behavior does not appear to be a problem for quality change applications. This conclusion comes with a caveat. Stated preference scenarios must include a status quo question. Without the status quo SP scenario in our model, a jointly estimated RP-SP model yields a trip estimate that is $25 \%$ larger and a willingness to pay per trip estimate that is over three times as large as we report in this paper. This suggests that trip overstatement may tend to 
occur in baseline forecasts of behavior and not in changes in forecast behavior as quality and other conditions change.

A second issue is that joint estimation of RP and SP data is often touted as a solution to hypothetical bias. We find that an independently estimated SP model performs just as accurately as the jointly estimated model. In addition, an independently estimated RP model combined with the univariate estimate of the change in trips between the SP status quo and SP beach width scenario would yield similar results as reported here.

Third, a typical concern with RP data is the inability to forecast beyond the range of historical experience. We find the models provide trip forecasts that align with the SP estimates. This result may be due to the wide range of beach widths at the southern NC beaches. Multiple site recreation demand with more limited variation in quality may not find results that are convergent valid.

Finally, our results illustrate that multi-site and single-site models can be usefully compared. Our SP elicitation focus was on aggregate trips in order to reduce the cognitive burden on survey respondents. At the time of the NC survey, obtaining multi-site SP data seemed like a difficult task for both the researcher and respondent. However, Jeon and Herriges (2005) subsequently report multiple-site SP and RP models that are convergent invalid. Further research to determine if multiple-site SP data lacks validity is needed.

Future research with these data might consider the beach access scenario. Yet, convincing tests of convergent validity using this scenario will be elusive. With the SP scenario we ask respondents for the number of beach trips they would take with a qualitative improvement in parking and beach access. Parking spaces is a quantitative measure in the RP models. The only comparison available is to estimate the additional trips and willingness to pay per trip with the 
SP data and then determine the number of parking spaces that leads to convergent validity with the multiple site RP data. An estimate of the number of additional parking spaces that is reasonable and convergent valid would be useful to policy makers. 


\section{References}

AAA. 2005. Personal communication. American Automobile Association, Heathrow, FL.

Azevedo, C. D., Herriges, J.A., and Kling, C.L. (2003) Combining revealed and stated preferences: consistency tests and their interpretations. American Journal of Agricultural Economics 85: 525-537.

Cameron and Trivedi. 1998. Regression Analysis of Count Data, Cambridge University Press.

Carson, Richard T., Nicholas E. Flores, Kerry M. Martin, and Jennifer L. Wright (1996) Contingent Valuation and Revealed Preferences Methodologies: Comparing the Estimates for Quasi-Public Goods. Land Economics 72:80-99.

Carson, Richard T., and W. Michael Hanemann. (2002) Contingent Valuation. Chapter 17 in Handbook of Environmental Economics: Volume 2 Valuing Environmental Changes, edited by Karl-Göran Mäler and Jeffrey R. Vincent, Elsevier North-Holland.

Egan, K. and J.A. Herriges (2006) Multivariate count data regression models with individual panel data from an on-site sample. Journal of Environmental Economics and Management 52:567-581.

Eiswerth, M.E., Englin, J., Fadali,E. and Shaw, W.D. (2000) The value of water levels in waterbased recreation: a pooled revealed preference/contingent behavior model, Water Resources Research, 36: 1079-1086.

Englin, J. and Cameron, T.A. (1996) Augmenting travel cost models with contingent behavior data. Environmental and Resource Economics 7: 133-147.

Freeman, A. M. (2003) The Measurement of Environmental and Resource Values. Resources for the Future Press. 
Grijalva, T., Bohara, A.K., Berrens, R.P. 2003 A Seemingly Unrelated Poisson Model for Revealed and Stated Preference Data. Applied Economics Letters 10: 443-446.

Grijalva, Therese, Robert P. Berrens, Alok K. Bohara, and W. Douglass Shaw (2002) Testing the Validity of Contingent Behavior Trip Responses. American Journal of Agricultural Economics 84(2): 401-414.

Haab, Timothy C., and Kenneth E. McConnell (2002) Valuing Environmental and Natural Resources: The Econometrics of Non-market Valuation. Northampton, MA: Edward Elgar.

Hanemann, W.M. (1978) A Theoretical and Empirical Study of the Recreation Benefits from Improving Water Quality in the Boston Area. Ph.D. Dissertation, Department of Economics, Harvard University.

Hellerstein, D., D. Woo, D. McCollum, and D. Donnelly (1993) ZIPFIP: A Zip and FIPS Database. Washington D.C.: U.S. Department of Agriculture, ERS-RTD.

Herstine, Jim, Jeffery Hill, Bob Buerger, John Whitehead and Carla Isom (2005) Determination of Recreation Demand for Federal Shore Protection Study Area: Overview and Methodology. Final Report Prepared for The U.S. Army Corps of Engineers, Wilmington District.

Jeon, Yongsik, and Joseph A. Herriges (2005) Convergent Validity of Contingent Behavior Responses in Models of Recreation Demand. Working Paper \#05031, Department of Economics Working Paper Series, Iowa State University.

Layman, R.C., Boyce, J.R., Criddle, K.R. (1996) Economic valuation of chinook salmon sport fishery of the Gulkana River, Alaska, under current and alternate management plans. Land Economics 72: 113-128. 
List, John A. and Craig A. Gallet (2001) What Experimental Protocol Influence Disparities Between Actual and Hypothetical Stated Values? Evidence from a Meta-Analysis. Environmental and Resource Economics 20:241-25.

Little, Joseph and Robert Berrens (2004) Explaining Disparities between Actual and Hypothetical Stated Values: Further Investigation Using Meta-Analysis. Economics Bulletin 3(6):1-13.

Parsons, George R. (2003) The Travel Cost Model. In A Primer on Nonmarket Valuation, Patricia A. Champ, Kevin J. Boyle, and Thomas C. Brown, eds., London: Kluwer. Parsons, George R., Paul M. Jakus and Ted Tomasi (1999) A Comparison of Welfare Estimates from Four Models for Linking Seasonal Recreational Trips to Multinomial Logit Models of Site Choice. Journal of Environmental Economics and Management 38:143-157.

Phaneuf, D.J., Kling, C.L., and J.A. Herriges (2000) Estimation and Welfare Calculations in a Generalized Corner Solution Model with an Application to Recreation Demand. Review of Economics and Statistics 82:83-92.

Phaneuf, Daniel J., and V. Kerry Smith (2005) Recreation Demand Models. Chapter 15 in Handbook of Environmental Economics: Volume 2 Valuing Environmental Changes, edited by Karl-Göran Mäler and Jeffrey R. Vincent, Elsevier North-Holland.

Phaneuf, Daniel J. and Chirs Siderelis (2003) An Application of the Kuhn-Tucker Demand Model to the Demand for Water Trail Trips in North Carolina. Marine Resource Economics 18:1-14.

von Haefen, R.H (2002) A complete characterization of the linear, log-linear, and semi-log incomeplete demand system models. Journal of Agricultural and Resource Economics 27:281-319. 
von Haefen, R.H. (2003) Incorporating Observed Choice into the Construction of Welfare Measures from Random Utility Models. Journal of Environmental Economics and Management 45:145-165.

von Haefen, R. and D.J. Phaneuf (2005) Kuhn-Tucker Demand System Approaches to Nonmarket Valuation. In Applications of Simulation Methods in Environmental and Resource Economics, A. Alberini and R. Scarpa, eds., Springer.

von Haefen, R.H., D.J. Phaneuf, and G.R. Parsons (2004) Estimation and Welfare Calculation with Large Demand Systems. Journal of Business and Economic Statistics 22:194-205.

Whitehead, John C., Timothy C. Haab and Ju-Chin Huang (2000) Measuring Recreation Benefits of Quality Improvements with Revealed and Stated Behavior Data. Resource and Energy Economics 22:339-354.

Whitehead, John C., Subhrendu K. Pattanayak, George L. Van Houtven and Brett R. Gelso (2008) Combining Revealed and Stated Preference Data to Estimate the Nonmarket Value of Ecological Services: An Assessment of the State of the Science. Journal of Economic Surveys 22:872-908.

Whitehead, John C. (2005) Environmental Risk and Averting Behavior: Predictive Validity of Revealed and Stated Preference Data. Environmental and Resource Economics 32:301316. 


\section{Appendix}

Welfare analysis and demand prediction in the KT model relies on Monte Carlo integration in which the unobserved heterogeneity (error) terms are drawn conditionally so that behavior at baseline travel cost and site conditions is replicated in the simulated outcomes. Given multiple simulated error vectors for each person compensating surplus is calculated for each error draw, and the average over people and draws provides an estimate of $E(C S)$.

Conditionally simulating the unobserved heterogeneity involves using the structure of the model and observation of each person's combination of interior and corner solutions for the available sites. For a particular person (suppressing the individual subscript $i$ ), how each element of the $J$-dimensional vector $\varepsilon$ is simulated depends critically on whether a site is visited. If the site $j$ is visited the structure of the model and the person's observed trips implies $\varepsilon_{j}=g_{j}(\cdot)$, where $g_{j}(\cdot)$ is the right hand side of (16). If site $j$ is not visited then equation (16) implies $\varepsilon_{j} \leq g_{j}(\cdot)$. In this case $\varepsilon_{j}$ can be simulated from a truncated type I extreme value distribution using the transformation

$$
\text { (A1) } \varepsilon_{j}=-\ln \left[-\ln \left(\exp \left(-\exp \left(-g_{j}(\cdot)\right)\right) U\right)\right] \text {, }
$$

where $U$ is a draw from a uniform distribution.

With values for the errors simulated all arguments of the general compensating surplus function in equation (14) are observed, and a computational device is needed to obtain CS conditional on an error draw. Von Haefen and Phaneuf (2005, p. 150) describe an efficient algorithm for this calculation that exploits the additive separability of the utility function in equation (15). Since it solves the expenditure minimization problem for each draw of the error the algorithm also provides predictions of changes in trip-taking behavior under changed site 
conditions. With this algorithm available the steps in the overall welfare calculation procedure for computing $E(C S)$ for a change in site characteristics can be summarized as follows:

1. On iteration $r$ simulate $\varepsilon_{i j}^{r}$ for $i=1, \ldots, N$ people and $j=1, \ldots, J$ sites where $\varepsilon_{i j}^{r}=g_{i j}(\cdot)$ if person $i$ visited site $j$ at baseline conditions, and $\varepsilon_{i j}^{r}$ is generated using (A1) if the site was not visited.

2. For each vector $\varepsilon_{i}^{r}=\left(\varepsilon_{i 1}^{r}, \ldots, \varepsilon_{i J}^{r}\right)^{\prime}$ compute $C S_{i}^{r}$ using the algorithm described by von Haefen and Phaneuf.

3. Upon completion of $r=1, \ldots, R$ iterations estimate the population $E(C S)$ using $E(C S)=R^{-1} \sum_{r=1}^{R} N^{-1} \sum_{i=1}^{N} C S_{i}^{r}$.

If standard errors on the estimate of $E(C S)$ or changes in trips taken are desired the three steps can be nested in a parametric or non-parametric bootstrap procedure. 
Table 1. Single Site Data Summary

\begin{tabular}{lccccc}
\hline \hline & Mean & & Std.Dev. & Minimum & Maximum \\
Revealed preference trips & 7.61 & 14.41 & & 0 & 100 \\
Stated preference trips & 10.01 & 16.58 & 0 & 123 \\
Stated preference trips with increased access & 13.91 & 24.23 & 1 & 200 \\
Stated preference trips with improved width & 11.20 & 19.9 & 1 & 200 \\
Typical trip travel cost (\$'s) & 104.82 & 67.1 & 1.03 & 305.07 \\
Household income (\$'s) & 58,436 & 27,160 & 10,000 & 100,000 \\
Married (=1) & 0.69 & - & 0 & 1 \\
Children (count) & 0.76 & 1.05 & 0 & 5 \\
Sample size $=419$ & & & & \\
\hline
\end{tabular}


Table 2. Multiple Site Data Summary

\begin{tabular}{|c|c|c|c|c|c|c|c|}
\hline County & $\underline{\text { Beach }}$ & $\begin{array}{l}\frac{\text { Trips }}{(\% \text { of }} \\
\underline{\text { total })}\end{array}$ & $\underline{\underline{\text { Mean }}} \underline{\underline{\text { Trips/Person }}}$ & $\underline{\text { Width }}$ & $\underline{\text { Public }}$ & $\frac{\text { Parking }}{\underline{\text { Spaces }}}$ & Length \\
\hline Carteret & Fort Macon & 1.69 & 0.13 & 90 & 2 & 602 & 1.4 \\
\hline Carteret & Atlantic Beach & 17.43 & 1.33 & 135 & 19 & 662 & 4.9 \\
\hline Carteret & Pine Knoll Shores & 1.82 & 0.14 & 110 & 6 & 195 & 4.8 \\
\hline Carteret & Indian Beach / Salter Path & 0.53 & 0.04 & 90 & 2 & 131 & 2.5 \\
\hline Carteret & Emerald Isle & 12.10 & 0.92 & 130 & 69 & 550 & 11.5 \\
\hline $\begin{array}{l}\text { Onslow- } \\
\text { Pender }\end{array}$ & North Topsail Beach & 7.21 & 0.55 & 82 & 42 & 929 & 9.7 \\
\hline $\begin{array}{l}\text { Onslow- } \\
\text { Pender }\end{array}$ & Surf City & 4.36 & 0.33 & 90 & 36 & 272 & 5.1 \\
\hline $\begin{array}{l}\text { Onslow- } \\
\text { Pender }\end{array}$ & Topsail Beach & 1.00 & 0.08 & 110 & 37 & 234 & 4 \\
\hline New Hanover & Wrightsville Beach & 18.59 & 1.42 & 160 & 45 & 1479 & 4.5 \\
\hline New Hanover & Carolina Beach & 10.44 & 0.79 & 185 & 26 & 452 & 2 \\
\hline New Hanover & Kure Beach & 1.47 & 0.11 & 130 & 20 & 223 & 2.8 \\
\hline New Hanover & Fort Fisher & 0.31 & 0.02 & 400 & 2 & 240 & 1.9 \\
\hline Brunswick & Caswell Beach & 0.66 & 0.05 & 80 & 12 & 103 & 2.8 \\
\hline Brunswick & Oak Island & 3.61 & 0.27 & 120 & 66 & 821 & 7.5 \\
\hline Brunswick & Holden Beach & 5.74 & 0.44 & 90 & 21 & 200 & 6.8 \\
\hline Brunswick & Ocean Isle Beach & 9.12 & 0.69 & 85 & 28 & 341 & 5.3 \\
\hline Brunswick & Sunset Beach & 3.92 & 0.30 & 115 & 34 & 260 & 1.2 \\
\hline
\end{tabular}

Sample size $=419$ (3190 observed trips) 
Table 3. Single Site Demand Models

\begin{tabular}{|c|c|c|c|c|c|c|c|c|}
\hline \multirow[b]{2}{*}{ Parameters } & \multicolumn{2}{|c|}{$\begin{array}{c}\text { RP Negative } \\
\text { Binomial }\end{array}$} & \multicolumn{2}{|c|}{ SP RE Poisson } & \multicolumn{2}{|c|}{ RP-SP RE Poisson } & \multicolumn{2}{|c|}{ RP-SP RE Poisson } \\
\hline & $\underline{\text { Coeff. }}$ & $\underline{\text { t-ratio }}$ & $\underline{\text { Coeff. }}$ & $\underline{\text { t-ratio }}$ & $\underline{\text { Coeff. }}$ & $\underline{\text { t-ratio }}$ & $\underline{\text { Coeff. }}$ & $\underline{\text { t-ratio }}$ \\
\hline Constant & 2.043 & 10.990 & 2.609 & 21.640 & 2.280 & 19.100 & 2.026 & 16.200 \\
\hline Travel cost & $\begin{array}{c}- \\
0.008\end{array}$ & -7.300 & -0.009 & -10.800 & -0.008 & -10.880 & -0.008 & -10.390 \\
\hline Income & 0.016 & 5.370 & 0.014 & 6.060 & 0.014 & 6.350 & 0.017 & 7.070 \\
\hline Married & $0 . \overline{221}$ & -1.270 & -0.402 & -3.200 & -0.373 & -2.990 & -0.240 & -1.830 \\
\hline Children & $\begin{array}{c}- \\
0.158\end{array}$ & -2.340 & -0.167 & -3.350 & -0.166 & -3.360 & -0.132 & -2.530 \\
\hline Access improvement & & & 0.329 & 16.240 & 0.329 & 16.240 & 0.329 & 16.240 \\
\hline Increase in beach width & & & 0.112 & 5.270 & 0.112 & 5.270 & 0.112 & 5.270 \\
\hline Stated preference & & & & & 0.274 & 11.670 & 0.581 & 11.540 \\
\hline SP x Married & & & & & & & -0.160 & -3.290 \\
\hline SP x Children & & & & & & & -0.042 & -1.980 \\
\hline SP x Travel Cost & & & & & & & 0.000 & -0.050 \\
\hline SP x Income & & & & & & & -0.003 & -3.410 \\
\hline Alpha & 1.426 & 12.464 & 0.989 & 15.656 & 0.976 & 15.765 & 0.976 & 15.761 \\
\hline Log-Likelihood & \multicolumn{2}{|c|}{-1221.820} & \multicolumn{2}{|c|}{-3524.500} & \multicolumn{2}{|c|}{-4855.670} & \multicolumn{2}{|c|}{-4827.100} \\
\hline Cases & \multicolumn{2}{|c|}{419} & \multicolumn{2}{|c|}{419} & \multicolumn{2}{|c|}{419} & \multicolumn{2}{|c|}{419} \\
\hline Periods & \multicolumn{2}{|c|}{1} & \multicolumn{2}{|c|}{3} & \multicolumn{2}{|c|}{4} & \multicolumn{2}{|c|}{4} \\
\hline \multicolumn{9}{|l|}{ Predictions } \\
\hline Baseline Trips & \multirow{2}{*}{\multicolumn{2}{|c|}{7.55}} & \multicolumn{2}{|c|}{9.92} & \multicolumn{2}{|c|}{7.54} & \multicolumn{2}{|c|}{7.57} \\
\hline Change in Trips & & & \multicolumn{2}{|c|}{1.17} & \multicolumn{2}{|c|}{0.89} & \multicolumn{2}{|c|}{0.90} \\
\hline Willingness to Pay & & & \multicolumn{2}{|c|}{$\$ 138$} & \multicolumn{2}{|c|}{$\$ 105$} & \multicolumn{2}{|c|}{$\$ 106$} \\
\hline
\end{tabular}


Table 4. Selected Count Data Demand System Results ${ }^{\mathrm{a}}$

\begin{tabular}{|c|c|c|c|c|c|c|c|c|}
\hline \multirow[b]{2}{*}{ Parameters } & \multicolumn{2}{|c|}{ Model 1} & \multicolumn{2}{|c|}{ Model 2} & \multicolumn{2}{|c|}{ Model 3} & \multicolumn{2}{|c|}{ Model 4} \\
\hline & $\underline{\text { Coeff. }}$. & $\underline{\text { t-ratio }}$ & $\underline{\text { Coeff. }}$ & $\underline{\text { t-ratio }}$ & $\underline{\text { Coeff. }}$ & $\underline{\text { t-ratio }}$ & Coeff. & $\underline{\text { t-ratio }}$ \\
\hline Constant & -1.42 & -1.54 & -0.98 & -0.26 & -1.30 & -0.40 & -3.35 & -0.97 \\
\hline Income & 0.02 & 4.95 & 0.02 & 4.95 & 0.02 & 4.98 & 0.02 & 5.04 \\
\hline Children & -0.22 & -2.68 & -0.22 & -2.67 & -0.22 & -2.69 & -0.21 & -2.70 \\
\hline Married & -0.17 & -0.91 & -0.18 & -0.94 & -0.18 & -0.93 & -0.20 & -1.09 \\
\hline Parking Spaces & 0.0007 & 1.82 & 0.0008 & 2.00 & 0.0008 & 1.86 & & \\
\hline \# Public Access & 0.02 & 1.28 & 0.02 & 1.36 & 0.02 & 1.91 & 0.03 & 1.79 \\
\hline Length & 0.0006 & 0.01 & -0.01 & -0.14 & & & 0.02 & 0.21 \\
\hline Width & 0.0012 & 0.17 & & & & & & \\
\hline Log(Width) & & & -0.06 & -0.08 & 0.00 & 0.00 & 0.46 & 0.66 \\
\hline Fort Macon & 0.39 & 0.53 & 0.31 & 0.43 & 0.34 & 0.47 & 0.76 & 1.07 \\
\hline Fort Fisher & -1.53 & -0.71 & -1.11 & -0.76 & -1.20 & -0.89 & -1.74 & -1.27 \\
\hline Alpha & 26.95 & 14.17 & 26.96 & 14.17 & 26.96 & 14.17 & 27.08 & 14.35 \\
\hline Log-Like & \multicolumn{2}{|c|}{-2668.55} & \multicolumn{2}{|c|}{-2668.57} & \multicolumn{2}{|c|}{-2668.58} & \multicolumn{2}{|c|}{-2669.86} \\
\hline Observations & \multicolumn{2}{|c|}{419} & \multicolumn{2}{|c|}{419} & \multicolumn{2}{|c|}{419} & \multicolumn{2}{|c|}{419} \\
\hline \multicolumn{9}{|l|}{ Predictions } \\
\hline Baseline Trips & \multicolumn{2}{|c|}{6.96} & \multicolumn{2}{|c|}{6.95} & \multicolumn{2}{|c|}{6.95} & \multicolumn{2}{|c|}{6.88} \\
\hline Change in Trips & \multicolumn{2}{|c|}{0.85} & \multicolumn{2}{|c|}{-0.254} & \multicolumn{2}{|c|}{0.004} & \multicolumn{2}{|c|}{2.16} \\
\hline Willingness to Pay & \multicolumn{2}{|c|}{$\$ 99$} & \multicolumn{2}{|c|}{$-\$ 29$} & \multicolumn{2}{|c|}{$\$ 0$} & \multicolumn{2}{|c|}{$\$ 270$} \\
\hline
\end{tabular}

${ }^{a}$ Robust standard errors used. Estimates for the 17 price parameters are shown in the Appendix. 
Table 5. Kuhn-Tucker Demand Results ${ }^{\mathrm{a}}$

\begin{tabular}{|c|c|c|c|c|c|c|c|c|}
\hline & \multicolumn{2}{|c|}{ Model 1} & \multicolumn{2}{|c|}{ Model 2} & \multicolumn{2}{|c|}{ Model 3} & \multicolumn{2}{|c|}{ Model 4} \\
\hline & $\underline{\text { Coeff. }}$ & $\underline{\text { t- }}$ & $\underline{\text { Coeff. }}$ & $\underline{\text { t-ratio }}$ & $\underline{\text { Coeff. }}$ & $\underline{\text { t-ratio }}$ & $\underline{\text { Coeff. }}$ & $\underline{\text { t-ratio }}$ \\
\hline Children & -0.03 & -0.56 & -0.03 & -0.56 & -0.03 & -0.57 & -0.03 & -0.57 \\
\hline Married & -0.01 & -0.06 & -0.01 & -0.07 & -0.01 & -0.08 & -0.01 & -0.06 \\
\hline Parking Spaces & 0.02 & 1.40 & 0.02 & 1.17 & 0.01 & 1.08 & & \\
\hline \# Public Access & -0.01 & -1.13 & -0.01 & -1.19 & 0.00 & -1.39 & 0.00 & -0.84 \\
\hline Length & 0.02 & 0.48 & 0.02 & 0.53 & & & 0.01 & 0.33 \\
\hline Width & 0.01 & 2.51 & & & & & & \\
\hline Log(Width) & & & 0.86 & 2.92 & 0.78 & 3.13 & 0.98 & 3.24 \\
\hline Fort Macon & 0.56 & 2.72 & 0.61 & 2.96 & 0.58 & 2.94 & 0.67 & 3.33 \\
\hline Fort Fisher & -1.10 & -1.53 & -0.47 & -1.04 & -0.38 & -0.91 & -0.63 & -1.37 \\
\hline$\theta$ & 1.57 & 4.16 & 4.95 & 3.39 & 4.51 & 3.77 & 5.41 & 3.61 \\
\hline$\rho *$ & -0.46 & -2.34 & -0.46 & -2.42 & -0.46 & -2.42 & -0.46 & -2.34 \\
\hline$\mu *$ & 0.24 & 6.52 & 0.24 & 6.51 & 0.24 & 6.52 & 0.24 & 6.52 \\
\hline Log-Like & \multicolumn{2}{|c|}{-2693.27} & \multicolumn{2}{|c|}{-2692.78} & \multicolumn{2}{|c|}{-2692.85} & \multicolumn{2}{|c|}{-2693.01} \\
\hline Fixed Effects & \multicolumn{2}{|c|}{ YES } & \multicolumn{2}{|c|}{ YES } & \multicolumn{2}{|c|}{ YES } & \multicolumn{2}{|c|}{ YES } \\
\hline Observations & \multicolumn{2}{|c|}{419} & \multicolumn{2}{|c|}{419} & \multicolumn{2}{|c|}{419} & \multicolumn{2}{|c|}{419} \\
\hline \multicolumn{9}{|l|}{ Predictions } \\
\hline Baseline Trips & \multicolumn{2}{|c|}{7.61} & \multicolumn{2}{|c|}{7.61} & \multicolumn{2}{|c|}{7.61} & \multicolumn{2}{|c|}{7.61} \\
\hline Change in Trips & \multicolumn{2}{|c|}{1.23} & \multicolumn{2}{|c|}{1.08} & \multicolumn{2}{|c|}{0.98} & \multicolumn{2}{|c|}{1.20} \\
\hline Willingness to Pay & \multicolumn{2}{|c|}{$\$ 374$} & \multicolumn{2}{|c|}{$\$ 311$} & \multicolumn{2}{|c|}{$\$ 279$} & \multicolumn{2}{|c|}{$\$ 354$} \\
\hline
\end{tabular}

${ }^{\mathrm{a}}$ Robust standard errors used. 
Table 6. Change in Trips and Willingness to Pay for Beach Width Scenario ${ }^{a}$

\begin{tabular}{|c|c|c|c|c|}
\hline & \multicolumn{2}{|c|}{ Multiple Site } & \multicolumn{2}{|c|}{ Single Site } \\
\hline & $\underline{\text { RP Count }}$ & $\frac{\text { RP-Kuhn }}{\text { Tucker }}$ & $\frac{\mathrm{RP}-\mathrm{SP}}{(\mathrm{SP}=0)}$ & $\frac{\mathrm{RP}-\mathrm{SP}}{(\mathrm{SP}=1)}$ \\
\hline Baseline Predicted Trips & $6.88(0.61)$ & $7.61(-)$ & $7.57(0.49)$ & $9.91(0.53)$ \\
\hline Change in Trips & $2.16(4.51)$ & $1.08(0.40)$ & $0.90(0.18)$ & $1.17(0.25)$ \\
\hline Annual Welfare Change & $\$ 270(842)$ & $\$ 309(115)$ & $\$ 106(22)$ & $\$ 126(92)$ \\
\hline
\end{tabular}

${ }^{\mathrm{a}}$ Standard errors in parentheses computed using 100 Krinsky-Robb replcations. 
Appendix Table: Full Count Data Demand Results

\begin{tabular}{|c|c|c|c|c|c|c|c|c|}
\hline & \multicolumn{2}{|c|}{ Model 1} & \multicolumn{2}{|c|}{ Model 2} & \multicolumn{2}{|c|}{ Model 3} & \multicolumn{2}{|c|}{ Model 4} \\
\hline & Coeff. & $\underline{\text { t-ratio }}$ & Coeff. & t-ratio & Coeff. & t-ratio & Coeff. & t-ratio \\
\hline Constant & -1.42 & -1.54 & -0.98 & $\overline{-0.26}$ & $\overline{-1.30}$ & $\overline{-0.40}$ & -3.35 & -0.97 \\
\hline Income & 0.02 & 4.95 & 0.02 & 4.95 & 0.02 & 4.98 & 0.02 & 5.04 \\
\hline Children & -0.22 & -2.68 & -0.22 & -2.67 & -0.22 & -2.69 & -0.21 & -2.70 \\
\hline Married & -0.17 & -0.91 & -0.18 & -0.94 & -0.18 & -0.93 & -0.20 & -1.09 \\
\hline Parking Spaces & 0.00 & 1.82 & 0.00 & 2.00 & 0.00 & 1.86 & & \\
\hline \# Public Access & 0.02 & 1.28 & 0.02 & 1.36 & 0.02 & 1.91 & 0.03 & 1.79 \\
\hline Length & 0.00 & 0.01 & -0.01 & -0.14 & & & 0.02 & 0.21 \\
\hline Width & 0.00 & 0.17 & & & & & & \\
\hline Log(Width) & & & -0.06 & -0.08 & 0.00 & 0.00 & 0.46 & 0.66 \\
\hline Fort Macon & 0.39 & 0.53 & 0.31 & 0.43 & 0.34 & 0.47 & 0.76 & 1.07 \\
\hline Fort Fisher & -1.53 & -0.71 & -1.11 & -0.76 & -1.20 & -0.89 & -1.74 & -1.27 \\
\hline $\mathrm{p} 1$ & -0.02 & -4.28 & -0.02 & -4.28 & -0.02 & -4.28 & -0.02 & -4.28 \\
\hline $\mathrm{p} 2$ & 0.00 & -1.29 & 0.00 & -1.17 & 0.00 & -1.38 & 0.00 & -0.97 \\
\hline p3 & -0.01 & -2.36 & -0.01 & -2.31 & -0.01 & -2.48 & -0.01 & -2.46 \\
\hline $\mathrm{p} 4$ & -0.02 & -4.45 & -0.02 & -4.48 & -0.02 & -4.48 & -0.02 & -4.44 \\
\hline p5 & -0.02 & -4.77 & -0.02 & -4.61 & -0.02 & -5.09 & -0.02 & -6.21 \\
\hline p6 & -0.03 & -4.52 & -0.03 & -4.50 & -0.03 & -4.66 & -0.03 & -4.54 \\
\hline p7 & -0.02 & -5.09 & -0.02 & -5.19 & -0.02 & -5.45 & -0.02 & -5.56 \\
\hline $\mathrm{p} 8$ & -0.03 & -5.06 & -0.03 & -5.10 & -0.03 & -5.31 & -0.03 & -5.38 \\
\hline p9 & -0.01 & -4.74 & -0.01 & -4.79 & -0.01 & -4.97 & -0.01 & -3.93 \\
\hline p10 & -0.01 & -2.61 & -0.01 & -2.60 & -0.01 & -2.60 & -0.01 & -3.16 \\
\hline p11 & -0.02 & -6.71 & -0.02 & -6.59 & -0.02 & -6.56 & -0.02 & -7.50 \\
\hline p12 & -0.02 & -2.71 & -0.02 & -2.71 & -0.02 & -2.71 & -0.02 & -2.71 \\
\hline p13 & -0.03 & -4.48 & -0.04 & -4.50 & -0.03 & -4.54 & -0.04 & -4.52 \\
\hline p14 & -0.03 & -7.16 & -0.03 & -7.24 & -0.03 & -7.49 & -0.03 & -7.49 \\
\hline p15 & -0.01 & -3.07 & -0.01 & -3.05 & -0.01 & -3.09 & -0.01 & -3.43 \\
\hline p16 & -0.01 & -3.86 & -0.01 & -3.91 & -0.01 & -3.95 & -0.01 & -3.91 \\
\hline $\mathrm{p} 17$ & -0.03 & -5.30 & -0.03 & -5.41 & -0.03 & -6.59 & -0.03 & -5.55 \\
\hline Alpha & 26.95 & 14.17 & 26.96 & 14.17 & 26.96 & 14.17 & 27.08 & 14.35 \\
\hline Log-Like & \multicolumn{2}{|c|}{-2668.55} & \multicolumn{2}{|c|}{-2668.57} & \multicolumn{2}{|c|}{-2668.58} & \multicolumn{2}{|c|}{-2669.86} \\
\hline Observations & \multicolumn{2}{|c|}{419} & \multicolumn{2}{|c|}{419} & \multicolumn{2}{|c|}{419} & \multicolumn{2}{|c|}{419} \\
\hline
\end{tabular}


Figure 1. Southern North Carolina Beaches

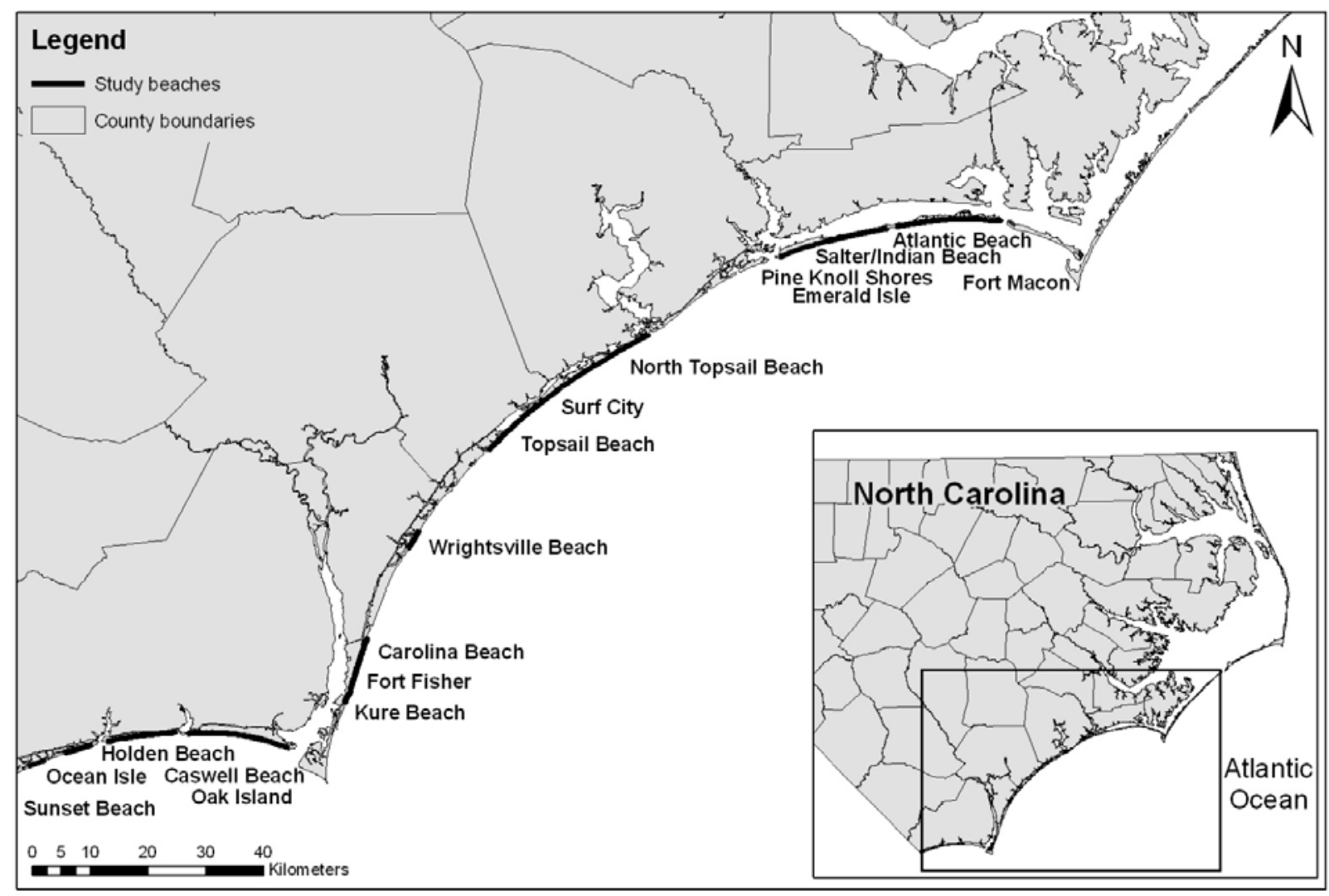

